\title{
CONSTRUCCIÓN DE UNA ESCALA PARA EVALUAR EL CONTEXTO FAMILIAR DESDE LA PERSPECTIVA DE LOS PADRES
}

\section{CONSTRUCTION OF A SCALE FOR ASSESSING FAMILY CONTEXT FROM THE PERSPECTIVE OF PARENTS}

\author{
María Cristina Richaud* y Elsa InÉs Bei ${ }^{* *}$
}

\begin{abstract}
*Doctora en Filosofía y Letras con orientación en Psicología. Miembro de la Carrera del Investigador del Consejo Nacional de Investigaciones Científicas y Técnicas (CONICET) en la categoría Superior y Directora del Doctorado en Psicología de la Universidad Católica Argentina (UCA).

**Profesora de Filosofía. Miembro de la Carrera del Personal de Apoyo a la Investigación, categoría Profesional Principal del Consejo Nacional de Investigaciones Científicas y Técnicas (CONICET). E-Mail: ebei@conicet.gov.ar
\end{abstract}

\section{RESUMEN}

Se presenta la construcción y validación de una escala que mide el contexto familiar desde la perspectiva de los padres, teniendo en cuenta la estimulación temprana que recibe el niño, y que Galvez (2007) define como la actividad de contacto con el bebé, que propicie sus potencialidades. Dentro de la línea ecológica sistémica de Bronfenbrenner (1987), Rodrigo y Palacios (1998) proponen tres aspectos del contexto familiar que afectan el desarrollo del niño: ideas y cogniciones de los padres con respecto al desarrollo, estilo de socialización y escenario e interacción educativa.

Una versión preliminar con 73 ítemes fue administrada a una muestra de 229 sujetos para estudiar sus propiedades psicométricas. Se examinó el poder discriminativo de los ítemes, mediante la prueba $t$ de diferencias de medias, resultando discriminativos 59 ítemes. Estos últimos fueron analizados factorialmente para determinar el número y la naturaleza de las dimensiones subyacentes. El análisis factorial exploratorio indicó la presencia de cuatro factores:ambiente familiar en relación a la estimulación personal, ambiente familiar con objetos estimulantes, ideas y creencias y prácticas de crianza. Los ítemes se agruparon guardando coherencia teórica con el planteo inicial. Se estudió la confiabilidad como consistencia interna, mediante el coeficiente Alpha de Cronbach obteniéndose un valor satisfactorio igual a .838 . A partir de los cuatro factores hallados se seleccionaron los 16 ítemes (cuatro por factor) con mayor pesaje, que conformaron la escala definitiva de tipo Likert que presentó buenos indicadores de fiabilidad, poder discriminativo de los ítemes y validez de constructo.

Palabras clave: Estimulación temprana; Propiedades psicométricas; Estilos de crianza; Creencias; Contexto familiar.

\section{ABSTRACT}

The purpose of this work is to present the construction and validation of a scale about ideas and beliefs that parents have respect the cognitive development of their children, the kind of breed adopted and the quality of the family context.

Among the different aspects that can be considered in what we call early relations, it is found the early stimulation that Galvez (2007) 
defines as the contact activity or play with a baby or child that propitiates, strengthens and develops appropriately its potentialities.

The family plays a fundamental role in the evolutional development of the child. Different models of family organization agreed in the recognition of its importance (Richaud de Minzi, 2005). It also greatly influences the organization of structural and material processes of the home, the planning of activities taking place there and how parents interact with the child (Muñoz Silva, 2005). This organization is determined in part by the parents' ideas and beliefs on the process of development of children and on the breeding practices (Siegel, McGillicuddy-Delisi \& Goodnow, 1992).

Taking into account an ecological systemic perspective, Bronfenbrenner's model (1987) shows the individual experience as a set of nearby structures, each inside the next, being the family the closest to the individual and which will offer the first stimulation experiences. From the point of view of the ecological theory of the individual's development, Rodrigo and Palacios (1998) have defined three family context aspects that will affect the child's development and have been taken as indicators for the construction of this instrument. These are the parents' ideas and beliefs regarding their children's cognitive development, the parent$n g$ style adopted by them and the quality of the family context.

The items proposed by the authors were selected and reformulated taking into account the direct observation of Argentine parents and presented in a Likert format. The preliminary scale remained constituted by 73 items presented in a Likert scale of three points. No agreement, Moderately agree, and Highly agree. Questions referred to the level of education of the parents, age, and quantity of offspring was also incorporated.

The preliminary scale was administered to 229 families of children attending four schools in the city of Buenos Aires (Argentina).

To study the psychometric fitness of the scale it was first examine the discriminative capacity of the items using $t$ test of mean differences or the contrasting group's method. The test was performed for the original 73 items, founding that 14 of them were no discriminatory. The preliminary scale was then reduced to 59 items. Factorial analysis of these 59 items was done, as a explo- ratory mode, to determine the number and nature of the dimensions present in the instrument.

This test indicated the presence of four factors: (a) family environment related to personal stimulation, (b) family environment which includes stimulating objects, (c) ideas and beliefs, and (d) breeding practices.

The items were grouped in coherence with the initial theoretical hypothesis. Based on factorial analysis the four heavier items in each factor $(>.40)$ that they were not factorially complex were taken into account to constitute the final version of the scale.

The factorization of the final 16 items clearly showed the items grouping in the four factors observed before (see Figure 2). These four factors explain $60.39 \%$ of variance (see Table 1 ).

Reliability as internal consistence of the scale of 16 reactive through the Cronbach Alpha was analyzed and a coefficient of .738 was obtained.

The scale here presented enables us to evaluate ideas and beliefs that parents have with respect to the evolving development of the child and how these materialize in its conduct related to the style of breeding as well as to the patterns of action and their interaction. The scale presents good psychometric properties, specially referring to the factorial structure being its reliability as its internal moderate consistency.

Key words: Early stimulation; Beliefs; Parenting style; Family context; Measurement.

\section{INTRODUCCIÓN}

Desde hace algunos años, la Psicología del Desarrollo ha puesto el acento en la importancia fundamental de las relaciones tempranas del niño con sus cuidadores. Entre los diferentes aspectos que pueden considerarse en lo que se denomina relaciones tempranas, se encuentra la estimulación temprana que Galvez (2007) define como la actividad de contacto o juego con un bebé o niño que propicie, fortalezca y desarrolle adecuada y oportunamente sus potencialidades. 
Dada la receptividad del niño a las influencias externas, especialmente a aquellas provenientes de sus cuidadores primarios, los estímulos bien dirigidos potencian el crecimiento y desarrollo del mismo. Desde este punto de vista, el desarrollo completo de las capacidades funcionales depende en gran parte de la experiencia sensorial temprana.

La familia desempeña un papel fundamental en el desarrollo evolutivo del niño. Diferentes modelos de organización familiar acuerdan en el reconocimiento de su importancia (Richaud de Minzi, 2005). La familia es durante los primeros años de vida del niño, su principal contexto de desarrollo y le proporciona el cuidado, afecto y apoyo necesarios para brindarle los elementos que le facilitan el conocimiento del mundo que lo rodea. También influye en gran medida la organización de los procesos estructurales y materiales del hogar, la planificación de las actividades que allí se llevan a cabo y cómo los padres interactúan con el niño (Muñoz Silva, 2005). Esta organización está determinada en parte por las ideas y creencias de los padres acerca del proceso de desarrollo de los niños y acerca de las prácticas de crianza (Sigel, McGillicuddy-Delisi \& Goodnow, 1992). Algunos autores diferencian entre estilos de crianza y prácticas de crianza, caracterizando a los primeros como actitudes y creencias y a las segundas, como los comportamientos específicos de los padres para guiar a sus hijos en el proceso de socialización. Sólis-Cámara Résendiz y Díaz Romero (2007) sostienen que si bien hay estudios que se refieren a un modelo integrador, en su análisis se enfocan sólo en el estilo de crianza, y proponen considerar a las prácticas de crianza -o guía general de los padres acerca de las cosas importantes para tener en cuenta al educar a sus hijos- como una concretización de sus creencias acerca de la misma. Chattás (2004) sostiene que el entorno en el que el niño ha sido criado es probablemente, el parámetro más importante en su desarrollo. Cada familia posee un perfil particular en cuanto a ideales y representaciones, pero los valores culturales de cada época ponen límites a la diversidad y canti- dad de modelos identificatorios. Por lo tanto, es necesario conocer y comprender la visión del mundo de la cultura en la que viven los niños y sus familias, que va a dar lugar a prácticas sociales habituales para esa cultura, ya que no existen patrones universalmente aceptados para la crianza.

La regularidad y estructuración de la estimulación que el niño recibe puede ser reconocida a partir de la descripción de las rutinas de la vida cotidiana: cómo se le presentan los objetos, cómo se alientan sus conductas exploratorias y su autonomía, cómo participan en actividades conjuntas que puedan promover su desarrollo cognitivo y lingüístico.

Desde una perspectiva ecológica sistémica, el modelo de Bronfenbrenner (1987) muestra la experiencia del individuo como un conjunto de estructuras próximas, cada una dentro de la siguiente, siendo la familia la más próxima al individuo y la que le va a ofrecer las primeras experiencias de estimulación.

Dentro de esta línea teórica, Rodrigo y Palacios (1998) proponen tres aspectos del contexto familiar que afectan el desarrollo del niño: (1) las ideas y cogniciones de los padres con respecto al desarrollo del niño, (2) el estilo de las relaciones interpersonales o estilo de socialización y (3) el escenario y la interacción educativa que se relaciona con el aspecto cognitivo.

\section{Calidad del ConteXto en el hogar}

Palacios (1987) por su parte, define tres clases de padres en función de sus creencias con respecto al desarrollo evolutivo de los niños:

1.- Padres modernos: dan importancia a la interacción con el medio, tienen expectativas evolutivas optimistas y sus prácticas de crianza priorizan la explicación y el diálogo.

2.- Padres tradicionales: tienen una creencia innatista del desarrollo. Para ellos el medio tiene poca influencia en el desarrollo de sus hijos, tienen expectativas 
evolutivas pesimistas y sus prácticas de crianza son coercitivas.

3.- Padres paradójicos: presentan ideas contradictorias, estando a veces cerca de los padres modernos y a veces, cerca de los tradicionales.

Estos estilos educativos en general se relacionan con prácticas de compromiso / soporte y de estimulación y aprendizaje o de indiferencia y poca estimulación para el aprendizaje. Así, los padres llamados modernos crean un ambiente favorable para el desarrollo del niño, mientras que los padres con ideas de tipo constructivista, cercanos a los modernos, generan un tipo de experiencias de aprendizaje más significativo, dándole al niño un papel más activo, pues tendrían una visión más compleja de su desarrollo (Muñoz Silva, 2005).

Con respecto al estilo de socialización, según Baumrind (1991)

"los padres son literalmente parte de uno mismo, en ambos sentidos, genéticamente y como resultado de los procesos de socialización..." (p. 60).

Baumrind desarrolló una tipología dimensional de crianza que combina interés o calidez y responsabilidad o control. A partir de sus entrevistas y observaciones iniciadas hacia 1967 propone tres estilos parentales diferentes que inciden en el desarrollo de las competencias cognoscitivas y sociales en el niño:

1.- Autoritativo o autorizado: son los padres afectuosos y sensibles a las necesidades del niño, pero firmes en el mantenimiento de normas y principios. Le otorgan libertad con normas claras que pueden cambiar si hay razones.

2.- Autoritario: Son los padres que proponen una disciplina estricta, con abundantes normas que generalmente no toman en cuenta las necesidades del niño ni son explicadas. Tienen escasas demostraciones de afecto y controlan más que no haya conductas indeseables, en vez de promover conductas deseables.

3.- Permisivo: son los padres que se caracterizan por el afecto y el dejar hacer. No hay normas y el niño no suele asumir compromiso. El niño no debe ser reprimido y hay que satisfacer sus demandas.

Hacia 1983 Maccoby y Martin completan el esquema de Baumrind diferenciando entre dos tipos distintos de crianza permisiva: los padres indulgentes y los padres negligentes. Los primeros se caracterizan por un bajo nivel de demanda pero un alto nivel de responsabilidad, son cálidos y tolerantes pero exigen pocas conductas maduras, ejercen poca autoridad y permiten a los niños autorregularse tanto como les sea posible, mientras que los negligentes tienen un bajo nivel de exigencia y de interés o responsabilidad, no se preocupan mucho por la conducta de sus hijos y a menudo están muy preocupados por sus propios problemas, descuidando sus responsabilidades como padres.

En trabajos posteriores, Baumrind (1991) utiliza también estas dos dimensiones: demandingness y responsiveness, y define demanda como los reclamos que hacen los padres a los niños relacionados con su integración en la familia, su madurez, su disciplina. A su vez, define capacidad de respuesta como aquellas acciones que tienen como finalidad fomentar la individualidad, la auto-regulación y la auto-afirmación, y que suponen apoyo y aprobación, teniendo en cuenta las necesidades del niño y sus demandas. Baumrind incorpora así este cuarto estilo parental propuesto a partir de esta diferenciación:

4.- Indiferente negligente: Se refiere a padres que muestran una mínima expresión de afecto, así como de respuesta a las necesidades del niño, que pueden llegar al abandono. Hay ausencia de normas y controles.

Si bien se ha considerado que el estilo autorizado predice resultados positivos en el 
desarrollo, algunos autores consideran que no siempre resulta el mejor cuando se tienen en cuenta distintos contextos socioeconómicos. Sin embargo, trabajos realizados con niños en vulnerabilidad social de distintas culturas muestran que, si bien varían los valores en las distintas dimensiones del estilo parental de acuerdo al contexto, el predominio de la aceptación con un control moderado siempre es mejor para el desarrollo del niño (Richaud, Mestre, Lemos, Tur, Ghiglione \& Samper, 2013).

Finalmente, la vida en el hogar con sus diferentes aspectos estructurales y materiales define el escenario educativo cotidiano.

Dada la importancia que tiene el contexto familiar para el crecimiento adecuado de los niños es importante contar con instrumentos de evaluación confiables del mismo.

En función de los aspectos que constituirían el contexto familiar tales como ideas y creencias de los padres con respecto al desarrollo del niño, estilo de socialización y escenario educativo, sería útil contar con una medida que abarque todos estos aspectos y que pueda reflejarse en un perfil integrador con respecto a qué ideas y creencias, qué estilo de socialización y qué estilo educativo informan los padres. Si bien existen distintos instrumentos, como entrevistas y cuestionarios, que evalúan separadamente los procesos mencionados, no existe un autorreporte que pueda ser respondido por padres y madres y que abarque todos los aspectos conjuntamente.

Por ello, el objetivo del estudio que se informa fue desarrollar una escala integradora acerca del contexto familiar, que presente buenas propiedades psicométricas.

\section{Metodología}

\section{CONSTRUCCIÓN DE LA ESCALA}

En primer lugar se seleccionaron los ítemes a incluir en la escala:

a.- Para operacionalizar las ideas y creencias de los padres con respecto al desa- rrollo de los hijos, se seleccionaron 19 ítemes de la versión abreviada del Cuestionario de Ideas para Padres y Madres de Palacios, Moreno y Muñoz-Tinoco (1991). Este cuestionario se administra en forma de entrevista estructurada y sus preguntas pueden ser clasificadas en 10 bloques, de los cuales sólo se tuvieron en cuenta para este trabajo los ocho siguientes: (1) información, (2) capacidades atribuidas al niño/a, (3) la contribución de la herencia y el medio, (4) relación madre-hijo durante el embarazo, (5) prácticas educativas generales: relaciones padres-hijo/a, (6) capacidad percibida para influir en el desarrollo, (7) atribuciones al comportamiento y (8) valores educativos.

Los reactivos seleccionados fueron adaptados al formato Likert.

Los dos bloques no incluidos están relacionados con el papel del padre y con ideas vinculadas a la acción. En el primer caso, el cuestionario abreviado incorpora una sola pregunta, en la que se dan opciones para elegir entre el padre o la madre o ambos. En el segundo caso, las preguntas se relacionan con la respuesta dada a la pregunta anterior de dicha escala y es de tipo abierta. Ambos casos no se tuvieron en cuenta por considerarlos poco viables para un formato Likert.

b.- En relación con los estilos de crianza se seleccionaron reactivos del Inventario de Conductas Parentales de Merino Soto, Díaz Casapía y DeRoma (2004). Este instrumento es la validación y tradución del Parental Behavior Inventory de Lovejov, Weis, O'hare y Rubin ( 1999), y esos reactivos se refieren a Hostilidad/ Coerción y a Compromiso / Soporte, de acuerdo al modelo de Baumrind antes mencionado. Si bien se cuenta con escalas argentinas sobre las relaciones percibidas de los hijos con respecto a su relación con los padres (Richaud de Minzi, 2002, 2007) éstas son respondidas por los hijos, mientras que el insrtrumento seleccionado propone conocer la perspectiva de los padres. 
c.- Para estudiar la calidad del contexto familiar, se partió del modelo de Caldwell y Braddley (1984) y se seleccionó el Cuestionario de la Vida Cotidiana (Moreno, Palacios \& González, 1989) que es una adaptación al español del HOME (Home Observation for Measurement of the Environment - Bradley \& Caldwell, 1977). Se trata de una entrevista estructurada que evalúa la calidad del contexto familiar tratando de considerar la estimulación y el apoyo que el niño recibe. Posee indicadores con respecto a aspectos relativos al entorno físico de la casa, otros que refieren a la interacción durante la entrevista y por último, aquellos que relatan experiencias vividas. Existen diferentes versiones de acuerdo con la edad del niño, ya que la misma determina cambios en las dimensiones a ser evaluadas. La entrevista abarca las siguientes dimensiones: (1) Materiales de estimulación para el aprendizaje, (2) Estimulación lingüística, (3) Orgullo - afecto - ternura, (4) Estimulación académica, (5) Modelado y estimulación de la madurez social, (6) Diversidad de experiencias y (7) Aceptación.

Los ítemes propuestos por los autores fueron seleccionados y reformulados en función de la observación directa de padres argentinos y presentados en un formato Likert.

El instrumento preliminar quedó constituido por 73 ítemes presentados en una escala Likert de tres puntos: Nada de acuerdo, Moderadamente de acuerdo y Muy de acuerdo. También se incorporaron preguntas referidas al nivel de estudio de los padres, edad y cantidad de hijos.

\section{PARTICIPANTES}

Los protocolos fueron entregados a los padres a través de los maestros, previa autorización de los directivos del colegio y en el caso de los establecimientos dependientes del Gobierno de la Ciudad de Buenos Aires, con el consentimiento, además, del supervi- sor del distrito escolar. Fueron completados anónimamente y respondidos sólo cuando el padre manifestó su voluntad de hacerlo. Se incluía una explicación de los aspectos a evaluar y el objetivo de estudio.

La escala preliminar fue administrada a 229 familias de niños que concurrían a cuatro escuelas de la ciudad de Buenos Aires: una privada de clase media profesional, dos de nivel socioeconómico medio bajo y una de clase socioeconómica media. La media de edad de los niños fue igual a 6.2 años, la de las madres, 36.41 años y la de los padres, 39.12 años. Con respecto a la escolaridad, un $35.4 \%$ de las madres tenía estudios universitarios completos, mientras que un $26.9 \%$ no había terminado la escuela secundaria, incluyendo en este valor un $13.9 \%$ que sólo tenía estudios primarios y un $4 \%$ que no había terminado la primaria. Con respecto a la escolaridad de los padres, un 31\% tenía estudios universitarios completos y un $30 \%$ no tenía estudios secundarios completos, incluyendo un $15.7 \%$ que sólo tenía estudios primarios y un $2.4 \%$ que no había completado la escuela primaria.

\section{ANÁLISIS DE LOS DATOS Y RESULTAdOS}

\section{ESTUDIO DE LOS ÍTEMES}

En primer lugar, para estudiar la adecuación psicométrica de la escala se examinó la capacidad discriminativa de los ítemes, mediante la prueba $t$ de diferencias de medias o método de grupos contrastantes.

Luego de hallar los valores totales para cada sujeto, éstos se reagruparon de mayor a menor de acuerdo con los valores obtenidos. Se trabajó con los cuartiles 25 y 75 que representaban los valores más bajos y más altos, respectivamente, de los puntajes y se calcularon las medias y los desvíos de cada reactivo en cada uno de los dos grupos. Se realizó la prueba $t$ de diferencias de medias para los 73 ítemes originales, encontrando que 14 de los mismos no resultaron discriminativos. La escala preliminar quedó entonces reducida a 59 ítemes. 
$\mathrm{Al}$ analizar los ítemes no discriminativos se encontró que siete de ellos correspondían a prácticas de crianza, de los cuales cinco se vinculaban a Hostilidad / Coerción y expresaban conductas que podían ser vistas con descrédito social y condicionar la respuesta (Richaud, 2010), como por ejemplo: "Lo castigo, le doy palmadas (chirlos), le tiro de los pelos o le pego", "Pierdo la paciencia cuando mi hijo/a no hace lo que le digo", "Le digo cosas que podrían hacerlo sentir mal". Con respecto a los otros ítemes no discriminativos, cinco se referían a la calidad del contexto familiar y tres de ellos correspondían a la dimensión Diversidad de experiencias, lo que permitiría pensar también en una respuesta condicionada por lo esperado. Enunciados como "El niño come por lo menos una comida al día con sus padres", o "El niño sale de la casa a pasear con algún familiar por lo menos cada 15 días", o "Le permitimos al niño/a elegir alimentos en el supermercado", suponen una interacción positiva con el niño, cuya inexistencia podría ser mal vista socialmente. Los otros dos reactivos que no discriminaron, expresan ideas y creencias de los padres y corresponden a las dimensiones de Información, en relación con la importancia que se le da a la interacción con el medio ("Es importante informarse con respecto a la crianza de un hijo/a") y Capacidades atribuidas al niño, con un ítem que expresa optimismo con respecto al calendario evolutivo del niño ("Antes de los dos años un niño/a es capaz de responder cuando se lo llama").

\section{ESTUDIO FACTORIAL}

Se realizó a modo exploratorio un análisis factorial de los 59 ítemes de la escala preliminar, para determinar el número y la naturaleza de las dimensiones presentes en el instrumento.

Se utilizó el método de componentes principales y rotación oblimin. Previamente se determinó si la matriz de correlaciones era factorizable a través de la prueba de esfericidad de Bartlett $\left(\chi^{2}=4077.138, p=.000\right)$, que indicó que la matriz de correlaciones era diferente de la matriz identidad, y con el índice Kaiser-Mayer-Olkin que resultó igual a .716, es decir un valor mediano, aceptable. Para la extracción de factores se tomó en consideración la prueba de sedimentación de Cattell que presenta gráficamente la magnitud de los autovalores (ver Figura 1). Esta prueba indicó la presencia de cuatro factores: (a) Ambiente familiar en relación a la estimulación personal, (b) Ambiente familiar que incluye objetos estimulantes, (c) Ideas y creencias y (d) Prácticas de crianza. Los ítemes se agruparon guardando coherencia teórica con el planteo inicial. En base al análisis factorial precedente se tomaron en consideración los cuatro ítemes que tuvieron los mayores pesajes en cada factor $(>.40)$ y que no eran factorialmente complejos, que constituyeron la versión definitiva de la escala.

ESTUDIO DE LOS ÍTEMES QUE RESULTAN SELECCIONADOS PARA LA VERSIÓN DEFINITIVA ESTUDIO FACTORIAL (VALIDEZ DE CONSTRUCTO)

Nuevamente se factorizaron los 16 ítemes seleccionados. Se calculó el índice de adecuación muestral con la escala configurada con estos ítemes y se obtuvo un valor $\mathrm{KMO}=.735$ y la prueba de esfericidad de Barlett mostró un $\chi^{2}=997.095$ y $p=.000$, resultando pertinente el análisis factorial. La matriz de factorización mostró claramente la agrupación de los ítemes en los cuatro factores antes observados (ver Figura 2), los cuales explicaron el $60.39 \%$ de la variancia (ver Tabla 1). Con respecto al planteo teórico inicial, el primer factor reunió reactivos que hacen referencia a Materiales para la estimulación del aprendizaje; el segundo factor agrupó dos ítemes que hacen referencia a Compromiso / Soporte, uno a Orgullo / Ternura y uno a Calidad del contexto familiar en lo que hace a la estimulación lingüística, que hipotéticamente indica un compromiso de parte del padre para involucrarse en la situación de aprendizaje del hijo. El tercer factor incluyó reactivos que evalúan las ideas y creencias en relación con las prácticas edu- 
cativas en general, capacidades atribuidas al niño e información. El cuarto factor reunió cuatro reactivos que evalúan la calidad del contexto familiar, pero en lo referente a Estimulación académica o cognitiva y Orgullo y afecto. En relación con los aspectos teóricos señalados, el correspondiente a Calidad del contexto familiar mostró claramente dos dimensiones que explican una parte importante de la variancia y que merecen ser consideradas por separado.

\section{ESTUDIO DE LA CONFIABILIDAD}

Se analizó la fiabilidad como consistencia interna de la escala de 16 reactivos mediante el coeficiente Alpha de Cronbach y se obtuvo un valor igual a .738. La puntuación de cada ítem osciló entre un valor de 2 (Muy de acuerdo) hasta un valor de 0 (Nada de acuerdo). El valor medio más alto lo obtuvo el ítem 14 ("Entre mi hijo/a y yo nos damos abrazos y/o besos") $(M=1.95)$ y la mayor variabilidad de la respuesta la obtuvo el ítem 7 ("Creo que se debe retar al niño/a desde que nace cuando hace algo que no debe") $(D E=.770)$. Con relación a cómo afectó cada reactivo a la consistencia interna, es justamente este último reactivo el que, al ser eliminado de la escala determinaría el máximo coeficiente de confiabilidad, un poco más alto que el total $(\alpha=.742)$.

Se analizó el coeficiente de fiabilidad de cada factor encontrado. El primero, que corresponde a los ítemes denominados Calidad / Material de estimulación, obtuvo un Alpha igual a .885. Los valores medios fueron parejos (entre 1.72 y 1.62) obteniendo la mayor variabilidad el reactivo "El niño tiene juguetes para ejercitar la motricidad fina" $(D E=.693)$. Si se elimina este reactivo, el coeficiente de confiabilidad alcanzaría un valor igual a .893 .

El segundo factor, denominado Compromiso-soporte / Orgullo-ternura presentó un Alpha mucho menor $(\alpha=.581)$. La media más baja la obtuvo el reactivo "Mi hijo y yo tenemos tiempo para jugar, hacer dibujos y otras cosas" $(M=1.61)$. Si se eliminara este ítem, el Alpha aumentaría a .674. Este reactivo, que de acuerdo con el planteo teórico, corresponde a la dimensión Compromisosoporte relacionado con Prácticas de crianza, implica una demanda de tiempo del padre, lo que lo hace un poco diferente a los otros reactivos del factor. Supuestamente el número de ítemes afectó al valor de Alpha que resultó poco satisfactorio.

Con respecto al tercer factor, Ideas y creencias de los padres, el Alpha de Cronbach fue igual a .636. La media más baja $(M=.81)$ correspondió al ítem "Creo que se debe retar al niño desde que nace cuando hace algo que no debe", mostrando la máxima variabilidad y el valor de Alpha aumentaría a .660 si se eliminara el reactivo "Creo que la experiencia previa y los recuerdos de la infancia inciden en las ideas que uno tiene con respecto a la educación de los niños", que tuvo la media más alta $(M=1.56)$ y la menor variación $(D E=.564)$.

El último factor, Calidad del contexto, tiene reactivos referidos a la Estimulación académica con una carga de Compromisosoporte, pues no se trata sólo de tener materiales para aprender, sino que también implica una actitud activa del padre. La consistencia interna de este factor obtuvo un coeficiente Alpha igual a .603. La mayor variabilidad la mostró el ítem "Animamos al niño a aprender discursos fijos" $(D E=.630)$ y la media más alta la obtuvo el reactivo "Trato de enseñarle cosas nuevas" $(M=1.92)$. La eliminación de cualquiera de los reactivos no aumentaría el índice de confiabilidad.

\section{Discusıón}

Teniendo en cuenta la importancia que tiene para el desarrollo del niño el contexto familiar y sus aspectos cognitivos (ideas y creencias), relacionales y ambientales, el objetivo de este estudio fue construir un autorreporte que integrara estas características para ser respondido por los padres y/o las madres. De esta manera se podría tener una visión y evaluación más compleja del con- 
texto en el que crece el niño, a través de un instrumento parsimonioso y de fácil administración.

A partir de las preguntas acerca de la incidencia que tiene la experiencia previa en nuestras ideas, se pueden reconocer padres que están más próximos a planteamientos ambientalistas - interaccionistas. Por otra parte, formular preguntas que se refieran a la edad de adquisición por parte del niño, de alguna destreza o logro, puede indicar que se está en presencia de padres que aceptan un calendario evolutivo más o menos precoz, pudiendo estar relacionado con factores generacionales o socio-educacionales relativos a la edad de los padres y a su nivel de educación.

La escala aquí presentada posibilita evaluar las ideas y creencias que tienen los padres con respecto al desarrollo evolutivo del niño y cómo éstas se materializan en su conducta, tanto en lo relativo al estilo de crianza por ellos adoptado, como a los patrones de acción según los cuales interactúan. La misma informa sobre: calidad de estimulación material, capacidades de estimulación personales, prácticas educativas y capacidades atribuidas a los niños. Presenta buenas propiedades psicométricas, especialmente en lo que se refiere a su estructura factorial, siendo su confiabilidad como consistencia interna moderada.

Sería importante estudiar el comportamiento de la escala en otras muestras de diferentes partes del país, así como analizar su estructura separadamente para padres y madres, teniendo en cuenta además las distintas edades de los padres y de los hijos. También aportaría al estudio de la validez realizar un estudio factorial confirmatorio poniendo a prueba el modelo de los cuatro factores. Finalmente sería interesante su utilización y análisis en otras culturas. 
Richaud y Bei

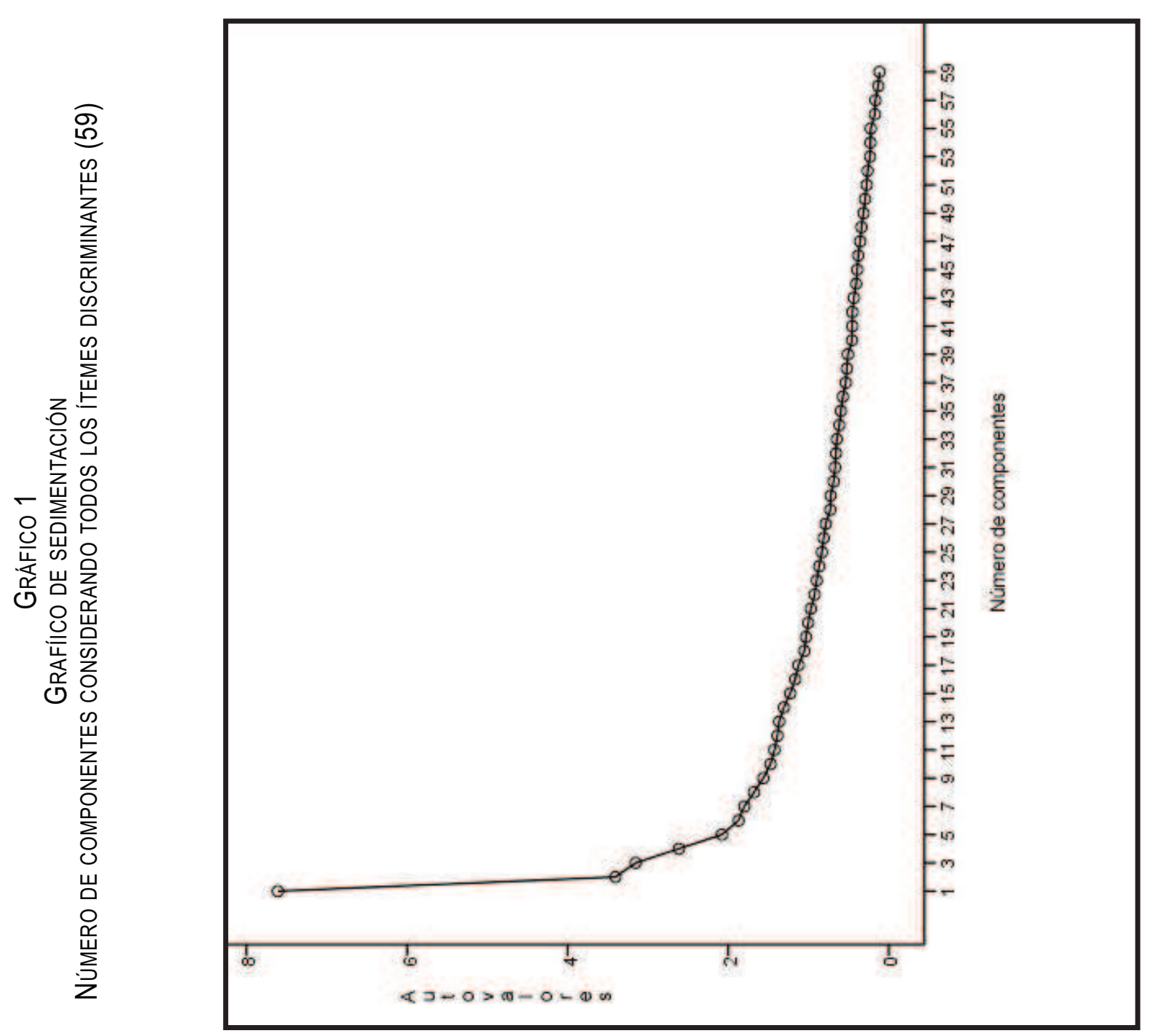







TABLA 1

ANÁLISIS FACTORIAL DE LA ESCALA DE CONTEXTO FAMILIAR POR EL MÉTODO DE COMPONENTES PRINCIPALES MATRIZ DE ESTRUCTURA

\begin{tabular}{|c|c|c|c|c|}
\hline \multirow[b]{2}{*}{ Itemes } & \multicolumn{4}{|c|}{ Componente } \\
\hline & 1 & 2 & 3 & 4 \\
\hline $\begin{array}{l}\text { 1.- El niño/a tiene juguetes para aprender nombres } \\
\text { de animales. } \\
\text { 2.- El niño/a tiene juguetes para el aprendizaje de } \\
\text { formas y tamaños. } \\
\text { 3.- El niño/a tiene juguetes para aprender los núme- } \\
\text { ros. } \\
\text { 4.- El niño/a tiene juguetes para ejercitar la motrici- } \\
\text { dad fina (pequeños, con detalles). } \\
\text { 5.- Acariciamos y besamos al niño/a muy a menudo. } \\
\text { 6.- Animamos a nuestro hijo/a a conversar y lo escu- } \\
\text { chamos. } \\
\text { 7.- Entre mi hijo/a y yo nos damos abrazos y/o } \\
\text { besos. } \\
\text { 8.- Mi hijo/a y yo tenemos tiempo para jugar, hacer } \\
\text { dibujos y otras cosas. } \\
\text { 9.- Creo que antes del año vale la pena comenzar a } \\
\text { explicar y razonar las cosas con los niños. } \\
\text { 10.- Vale la pena empezar a explicar y razonar las } \\
\text { cosas con los niños/as aunque todavía no entien- } \\
\text { dan. } \\
\text { 11.- Creo que se debe retar al niño/a desde que } \\
\text { nace cuando hace algo que no debe. } \\
\text { 12.- Creo que la experiencia previa y los recuerdos } \\
\text { de la infancia inciden en las ideas que uno tiene con } \\
\text { respecto a la educación de los niños. } \\
\text { 13.- Animamos al niño/a a aprender los números. } \\
\text { 14.- Animamos al niño/a a aprender discursos fijos } \\
\text { (canciones poesías, etc). } \\
\text { 15.- Animo a mi hijo/a a demostrar alguno de sus lo- } \\
\text { gros. } \\
\text { 16.- Trato de enseñarle cosas nuevas. }\end{array}$ & $\begin{array}{l}.887 \\
.887 \\
.871 \\
.794\end{array}$ & $\begin{array}{l}.806 \\
.742 \\
.717 \\
.492\end{array}$ & $\begin{array}{l} \\
\\
.773 \\
.739 \\
.727 \\
.470\end{array}$ & 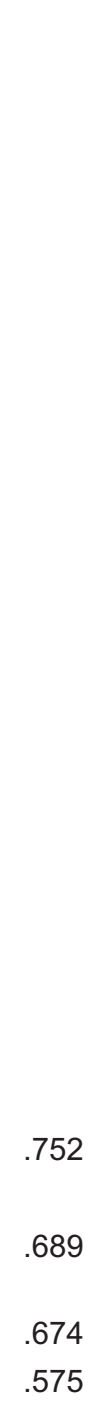 \\
\hline $\begin{array}{l}\% \sigma^{2} \text { explicada } \\
\alpha\end{array}$ & $\begin{array}{c}20.14 \\
.885\end{array}$ & $\begin{array}{l}13.99 \\
.581\end{array}$ & $\begin{array}{l}12.76 \\
.636\end{array}$ & $\begin{array}{r}13.49 \\
.603\end{array}$ \\
\hline
\end{tabular}

Nota

Método de rotación: Oblimin con Kaiser ( $N=229$ sujetos) 


\section{REFERENCIAS BIBLIOGRÁFICAS}

Baumrind, D. (1991). The influence of parenting style on adolescent competence and substance use. Journal of Early Adolescence, 11(1), 5695. http://dx.doi.org/10.1177/0272431691111 004

Bradley, R.H. \& Caldwell, B. (1977). Home observation for measurement of environment: A validation study of screening efficiency. American Journal of Mental Deficiency, 81 (5), 417-420.

Bronfenbrenner, U. (1987). La ecología del desarrollo humano [The ecology of the human development]. Barcelona: Paidós.

Caldwell, B. \& Braddley, R. (1984). Home observation for measurement of the environment. Recuperado el 23 de Marzo de 2010 de http:// www. bilbao.net/castilla/educacion/apoyo_e ducacion/rendimiento_academico

Chattás, A.J. (2004). Estilos de crianza [Styles of upbringing]. Recuperado el 5 de Marzo de 2013 de www.sap.org.ar/pronap.php

Galvez, L. (2007). Estimulación [Stimulation]. Recuperado el 8 de Mayo de 2012 de www. estimulacionenbebes.blogspot.com/2007/04/d efinición.html

Lovejov, M.C., Weis, R., O'hare, E. \& Rubin, E.C. (1999). Development and initial validation of the Parental Behavior Inventory. Psychological Assessment, 11(4), 534-545.

Maccoby, E.E. \& Martin, J.A. (1983). Socialization in the context of family: Parent-child interaction. En P.H. Mussen \& E.M. Hetherington, Handbook of child psychology. Vol. 4: Socialization, personality, and social development (pp. 1-100). New York: Wiley.

Merino Soto, C., Diaz Casapía, M. \& DeRoma, V. (2004). Validación del Inventario de Conductas Parentales: Un análisis factorial confirmatorio [Validation of the inventory of parenting behavior: An analysis factorial confirmatory]. Persona, 7, 145-162.

Moreno, M., Palacios, J. \& González, M. (1989). Cuestionario de la Vida Cotidiana [Question- naire of the daily life]. Trabajo no publicado. Sevilla, España: Universidad de Sevilla.

Muñoz Silva, A. (2005). La familia como contexto de desarrollo infantil. Dimensiones de análisis relevantes para la intervención educativa y social [Family as context of child development. Main components to educational and social intervention]. Portularia, V(2), 147163.

Palacios, J. (1987). Contenidos, estructura y determinantes de las ideas de los padres. Una investigación empírica [Contents, structure and determinants of the ideas of the parents. An empirical investigation]. Infancia y Aprendizaje, 39-40, 113-136.

Palacios, J., Moreno, M.C. \& Muñoz-Tinoco, V. (s.f.). Cuestionario de Ideas para Padres y Madres (versión abreviada) [Questionnaire of ideas for parents and mothers (brief version)]. España: Universidad de Sevilla.

Richaud de Minzi, M.C. (2002). Inventario acerca de la Percepción que tienen los Niños y las Niñas de las Relaciones con sus Padres y Madres. Versión 4 a 6 años [Inventory about the perception that the children and the girls have of the relationships with his parents and mothers. Version 4 to 6 years]. Revista Interamericana de Psicología, 36(1-2), 149- 164.

Richaud de Minzi, M.C. (2005). Estilos parentales y afrontamientos en niños [Parenting styles and coping in children]. Revista Latinoamericana de Psicología, 37(1), 47-58.

Richaud de Minzi, M.C. (2007). Inventario de Percepción de Estilos Parentales en Niños de 8 a 12 años [Perception inventory of parenting styles in children from 8 to 12 years]. Revista Iberoamericana de Diagnóstico y Evaluación Psicológica, 23(1), 63-81.

Richaud, M.C. (2010). Construcción de tests psicológicos [Construction of psychological tests]. Buenos Aires: CIIPME-CONICET.

Richaud, M.C., Mestre, M.V., Lemos, V., Tur, A., Ghiglione, M. \& Samper, P. (2013). La influencia de la cultura en los estilos parentales en contexto de vulnerabilidad social [The culture influence in the parenting styles in 
context of social vulnerability]. Avances en Psicología Latinoamericana, 21(2), 419-431.

Rodrigo, M.J. \& Palacios, J. (1998). Familia y desarrollo humano [Family and human development]. Madrid: Alianza Editorial.

Sigel, I., McGillicuddy-Delisi, A. \& Goodnow, J. (1992). Introduction to the second edition. En I. Sigel, A. McGillicuddy-Delisi \& J. Goodnow (Eds.), Parental beliefs in the zone proximal development: The role of parents (pp. XIIIXX). New Jersey: Erlbaum.

Sólis-Cámara Reséndiz, P. \& Díaz Romero, M. (2007). Relaciones entre creencias y prácticas de crianza de padres con niños pequeños [Relations between beliefs and practices of upbringing of parents with small children]. Anales de Psicología, 23(2), 177-184.

Centro Interdisciplinario de Investigaciones en Psicología Matemática y Experimental "Dr. Horacio J. A. Rimoldi" (CIIPME) Consejo Nacional de Investigaciones Cientificas y Técnicas (CONICET) Ciudad Autónoma de Buenos Aires República Argentina

Fecha de recepción: 20 de mayo de 2013 Fecha de aceptación: 21 de octubre de 2013 